\title{
HYDRODYNAMIC PERFORMANCE OF THE 3D HYDROFOIL AT THE COUPLED OSCILLATING HEAVE AND PITCH MOTIONS
}

\author{
ABBASI A.R. ${ }^{1}$, GHASSEMI H. ${ }^{1}$, HE, G. ${ }^{2}$ \\ ${ }^{1}$ Department of Maritime Engineering, Amirkabir University of Technology, Tehran, \\ Iran,e-mail:a.r.abbasi@aut.ac.ir,gasemi@aut.ac.ir \\ ${ }^{2}$ School of Ocean Engineering, Harbin Institute of Technology, Weihai, \\ China,e-mail: ghhe@hitwh.edu.cn
}

\begin{abstract}
The purpose of this paper is to study on the power extraction of the hydrofoil by performance of the coupled oscillating heave and pitch motions. The numerical analysis is conducted by using the Reynolds Average Navier-Stokes (RANS) equations and the realizable k- $\varepsilon$ turbulent model of the Star-CCM+ software. A 3D oscillating hydrofoil of NACA0015 section with aspect ratio of 7 is selected for the present analysis at two inflow velocities and three frequencies. The numerical results of lift and drag coefficients, horizontal and vertical forces coefficients, power efficiency in time domain and average value of those parameters are presented and discussed. According to the numerical results, the high efficiency of hydrofoil is found at the reduced frequency of 0.18 and the flow velocity of $1 \mathrm{~m} / \mathrm{s}$ and the low efficiency is obtained at the reduction frequency of 0.06 and the flow velocity of $2 \mathrm{~m} / \mathrm{s}$. Moreover, the contour results of vorticity, streamline and pressure distribution are also presented and discussed. The computational model depicts clear vortex patterns surrounding the hydrofoil, which has a major impact on the power performance of oscillating hydrofoil.
\end{abstract}

KEYWORDS: Hydrodynamic performance, Heave and pitch motions, Lift and drag, Reduced frequency, Power production.

\section{Introduction}

Ocean creatures including dolphins and whale, thanks to their evolved fins and tails, have fast maneuverability and strong propulsive performance. Many of these animal bodies strongly resemble high aspect ratio foils. Deep-term biomimetic experiments and animal findings have motivated people to improve current propulsive devices. Oscillating hydrofoil propellers demonstrate possible advantages in certain ways in contrast with conventional propellers [1]. The hydrofoils have more applications in the field of energy harvesting and marine renewable energy including Vertical Axis Turbine (VAT) [2] and Horizontal Axis Turbine (HAT) [3, 4]. Fig.1 shows several oscillating hydrofoils with similar performance and different views. As can be seen in Figure the mechanism of oscillating hydrofoil system is simple. The structure of the oscillating hydrofoil system contained from an arm and a hydrofoil that the hydrofoil has coupled heave and pitch motion when the arm has an angular motion around its axis at the end of the arm. Flapper wings are typical modes of propulsion used for raising or thrusting by most aquatic animals. However, because of the complex interactions between fluid and structures, the process of force generation utilizing flapping hydrofoils that vary from classic fixed-wing hydrodynamics is complicated and yet undefined [5,6]. The mechanics of the flapping hydrofoil was thoroughly studied using three forms of flaps, pure pitching motion, pure heaving motion, and mixed action, including the two pitching and heaving movements [7]. This Hydrofoils have a major impact to improve the performance of the marine vessel propulsion system and can reduce the total resistance [8]. The analysis of pitch and heave oscillating hydrofoils has expanded in recent years. In a variety of applications, flapping techniques have proved 
effective, including the production of renewable energy through tidal and wind flows, lifting generation in micro-aerial vehicles and the development of thrust for underwater motor systems [9]. Experiments were conducted to investigate the effects of amplitude and depth on drag decrement of a NACA0015 hydrofoil immersion for a frequency spectrum close to a free surface. Naval vessels are presently being requested to go faster, more efficiently. To accomplish this, some type of drag reduction would need to be used. However, these innovations have shown great potential for the drag reduction at low speeds and for stationary surfaces, they are not known for realistic conditions where the vessel movement is high speed and unstable. The most significant challenge was highlighted by unstable flows causing the break-up of fluid layers leading to large increases in drag. A drag reduction strategy that is technically feasible would be indifferent to vessel movement [10]. Martin, et al. developed a CFD simulation to investigate the influence of the ship hull wake on the hydrodynamic performance of a 2D and 3D hydrofoil at the combined oscillating heave-pitch motions [11]. Thaweewat, et al. used the boundary element approach to conduct a numerical analysis on a semi-active oscillating hydrofoil [12]. Within a wide variety of working conditions, an extremely high efficiency was discovered for such a design. They also stated that the openwater performances of a semi-active oscillating hydrofoil are comparable to variable-pitch turbines. McKinney et al. conducted the first work on an oscillating hydrofoil in 1981. In a wind tunnel, they tested a version of $90 \mathrm{~W}$. With a motion phase of $90^{\circ}$ a maximum power extraction output was achieved while a maximum power extraction phase of $110^{\circ}$ was achieved. A prototype obtained a 16.8 percent power extraction performance, a $30^{\circ}$ pitch amplitude, a 0.3 chord length amplitude and a decreased frequency of 0.12 [13]. Kinsey et al. worked in some subject of oscillating hydrofoils including prototype testing of a hydrokinetic turbine based on oscillating hydrofoils [14], Parametric study of an oscillating hydrofoil in a power-extraction regime [15], optimal operating parameters for an oscillating foil turbine at Reynolds number 500,000 [16] and computational fluid dynamics analysis of a hydrokinetic turbine based on oscillating hydrofoils [17]. Other energy-related works are the experimental parametric investigation of Tidal In-Stream Energy Converters (TISECs) that conducted by Huxham et al. They were focused on oscillating foil energy converter (OFEC). This type of device is manipulated passively or actively by one or several hydrofoils in order to generate lifting forces to move an oscillating heave of the foils. This heaving movement can be connected to a generator to collect the flow's electricity [18]. Generating controllable velocity fluctuations using twin oscillating hydrofoils conducted by Harding et al. In this research, an experimental system has been designed to control the instantaneous flow velocity in a water canal independently. The results of this model have been described for the simplified cases of oscillatory velocity perturbations in the vertical and directions of stream-wise distinctly [19]. Shimizu et al conducted an optimization analysis on an oscillating hydrofoil notion. The hydrofoil is supported elastically in the heavy direction with its semi-passive concept, while pitching movement is imposed. They used an evolutionary algorithm to improve operating conditions, to approximate aerodynamically and flow response forces [20]. Simpson was studying the performance of the oscillating hydrofoil power extraction in a towing tank in $\mathrm{Re}=$ 13,800 experimentally. The hydrofoil that they used in their study was NACA0012. They enforced a sinusoidal timing and height function in order to obtain an effective sinusoidal angle for $\alpha(t)$ progression. Wake patterns have been categorized for various heave amplitude values in the parametric frequency space and the maximum efficient AoA. The wake pattern mapping is recorded to match between different amplitudes when the frequency is normalized as the reduced frequency. If the Strouhal number is then used, this match is not observed [21]. The Reynolds number dependency of such a shape suggested by experimental results motivated to conduct another study using both numerical simulation and experimental data [22]. For a coupled heave and pitch oscillating hydrofoil, heave motion contributes to most of the thrust 
production, although an appropriate pitching angle and resulting operative can improve the propulsive efficiency to a wide-ranging [23]. Dewey et al. conducted the research about the unsteady tandem hydrofoil's propulsive performance in a side-by-side configuration. In their research, the time-averaged thrust creation and power input to the fluid are found to relate to both the oscillation phase differential and the transverse spacing between the foils. For oscillations at middle phase differentials, the first hydrofoil experiences an efficiency improvement and thrust while the other experiences a reduction in thrust and efficiency [24]. A three-dimensional wake impingement model and applications on tandem oscillating hydrofoils conducted by He et al. In this study, a wake impingement model was developed to distract wake zone to slip over the body surface, model the vortex-body interaction, and predict forces on fluctuating mechanisms. The model was also tested on oscillating hydrofoils configurations in tandem with an existing panel method code. Study results with the wake impingement model are shown to be in closer contract with limited published experimental data than those without the model [25]. Experimental and numerical analysis of the passive adaptive composite hydrofoil to decrease its lifting reaction to fluid load passively conducted by Giovannetti, et al. The goal of their work is to identify the possibility of structures to help control the lift generated by hydrofoils on some marine vessel [26]. The flapping foil power generation device has been investigated as a new form of mechanism for extracting energy [27]. Effect of wake interaction on the response of two tandem oscillating hydrofoils is another research conducted by Ma, et al. that in their research using a hydraulic system couples two hydrofoils and achieves the complete motion of flow-induced oscillating. Two hydrofoils have a tandem three-dimensional configuration because in such an arrangement permits the hydrofoil to obtain higher performance. They were found that the energy extraction efficiency and heaving amplitude of the upstream hydrofoil is more than that of the downstream hydrofoil because the downstream hydrofoil operates in the upstream hydrofoil wake [28]. He et al carried out an OpenFOAMbased model was established to investigate the influence of the effective AoA on the energy extraction efficiency [29]. The coupled heave and pitch motions of the 2D hydrofoil presented by using BEM carried out by Pourmostafa et al. Pressure distribution, lift and drag coefficients are presented and discussed at different conditions [30].

In this research, the oscillating hydrofoil with a couple motion of heave and pitch is numerically investigated in three reduced frequency of $0.06,0.12$, and 0.18 respectively and the flow velocity that used in this study is $1 \mathrm{~m} / \mathrm{s}$ and $2 \mathrm{~m} / \mathrm{s}$. Also, the turbulent model that used in this work is realizable $\mathrm{k}-\varepsilon$ that will be explained in other section of the paper. Table 1 shows the geometrical parameters of the hydrofoil.

Table 1. Geometrical parameters of hydrofoil

\begin{tabular}{cc}
\hline Parameters & Value \\
\hline Hydrofoil section & NACA0015 \\
Chord length $(\mathrm{c})$ & $0.24 \mathrm{~m}$ \\
Span $(\mathrm{b})$ & $1.68 \mathrm{~m}$ \\
Pitching axis & $\mathrm{c} / 3$ \\
Heaving amplitude $\left(H_{0}\right)$ & $\mathrm{c}$ \\
Pitching amplitude $\left(\theta_{0}\right)$ & $75^{\circ}$ \\
Motion phase $(\phi)$ & $90^{\circ}$ \\
\hline
\end{tabular}




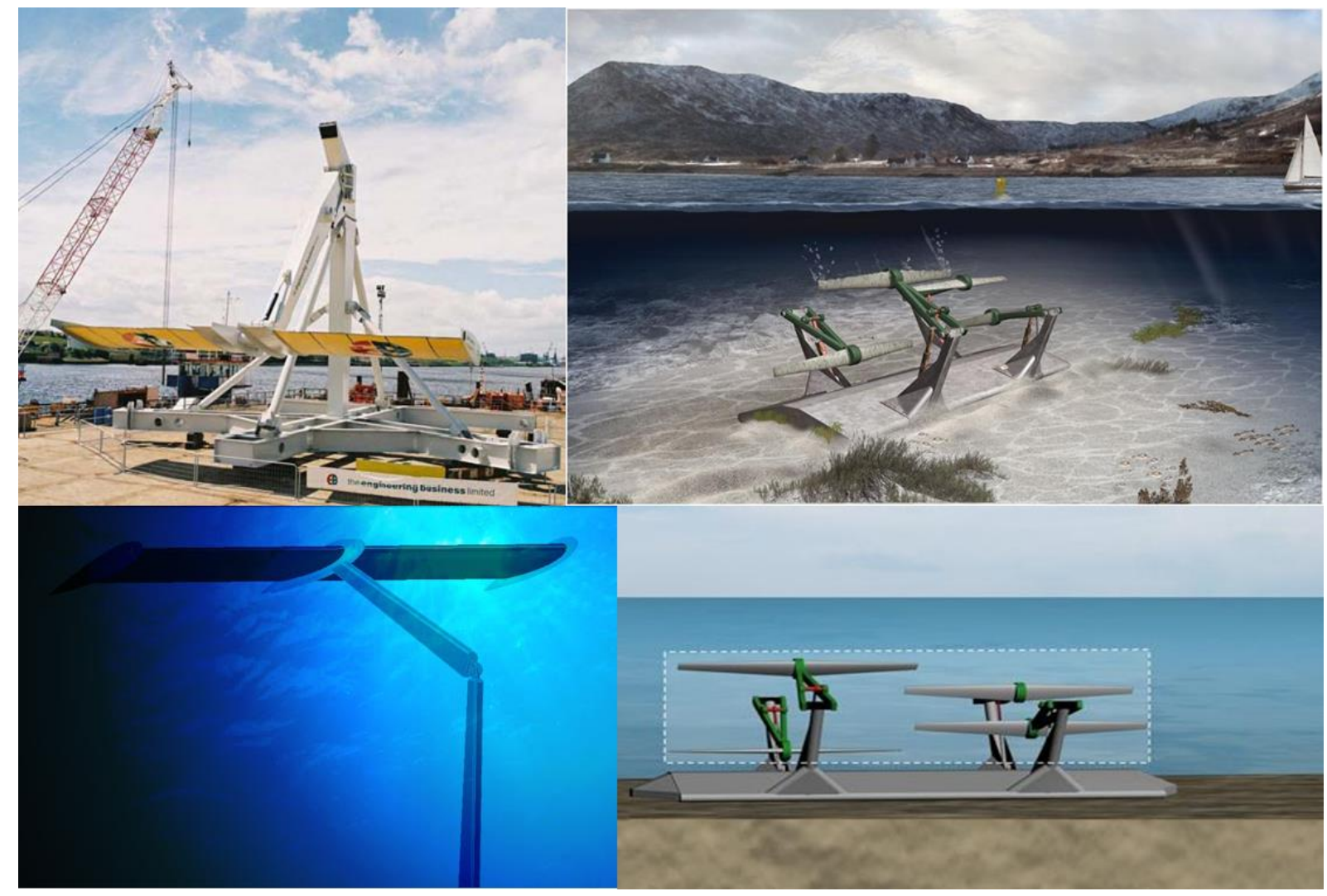

Fig. 1 Oscillating hydrofoils

\section{Boundary conditions and mesh generation}

Mesh acts as a point generator for the cycle of measurement. Calculating the distribution of the entity on increasing geometry level [31]. To draw the geometry and create the computational meshes for the hydrofoil and the external domain in this research adopted the software Star$\mathrm{CCM}+$. All the grids are structured with only trimmed cell mesh and this research is conducted based on the overset mesh method. The computational domain is divided into various parts: the external domain including the velocity inlet, pressure outlet, and symmetry plane, and internal domain including overset zone and hydrofoil, as shown in Fig.2. In this research, to apply mesh in the computational domain with the aim of simulating correct turbulent flow at the upstream, downstream and around the hydrofoil, various computational dimensions have been tried tested. Finally, the dimension of the computational domain is obtained as follows:

- the distances of hydrofoil center from inlet plane is $4.5 \mathrm{~b}$,

- the distances of hydrofoil center from outlet plane is $6.2 b$,

- the height (Y-axis) is $10 \mathrm{~b}$,

- $\quad$ the width (Z-axis) of the domain is $5.7 \mathrm{~b}$,

The parameters of $b$ and $c$ are the span and chord of the hydrofoil, respectively. Hydrofoil is settled in the middle of the Y-Z plane.

The mesh size is not uniform and created the smallest elements in the nearness of the hydrofoil surface due to the attendance of the boundary layer. Fig. 3 and Fig. 4 are shown all of the mesh domain around the hydrofoil and mesh of hydrofoil surface with details, respectively. 


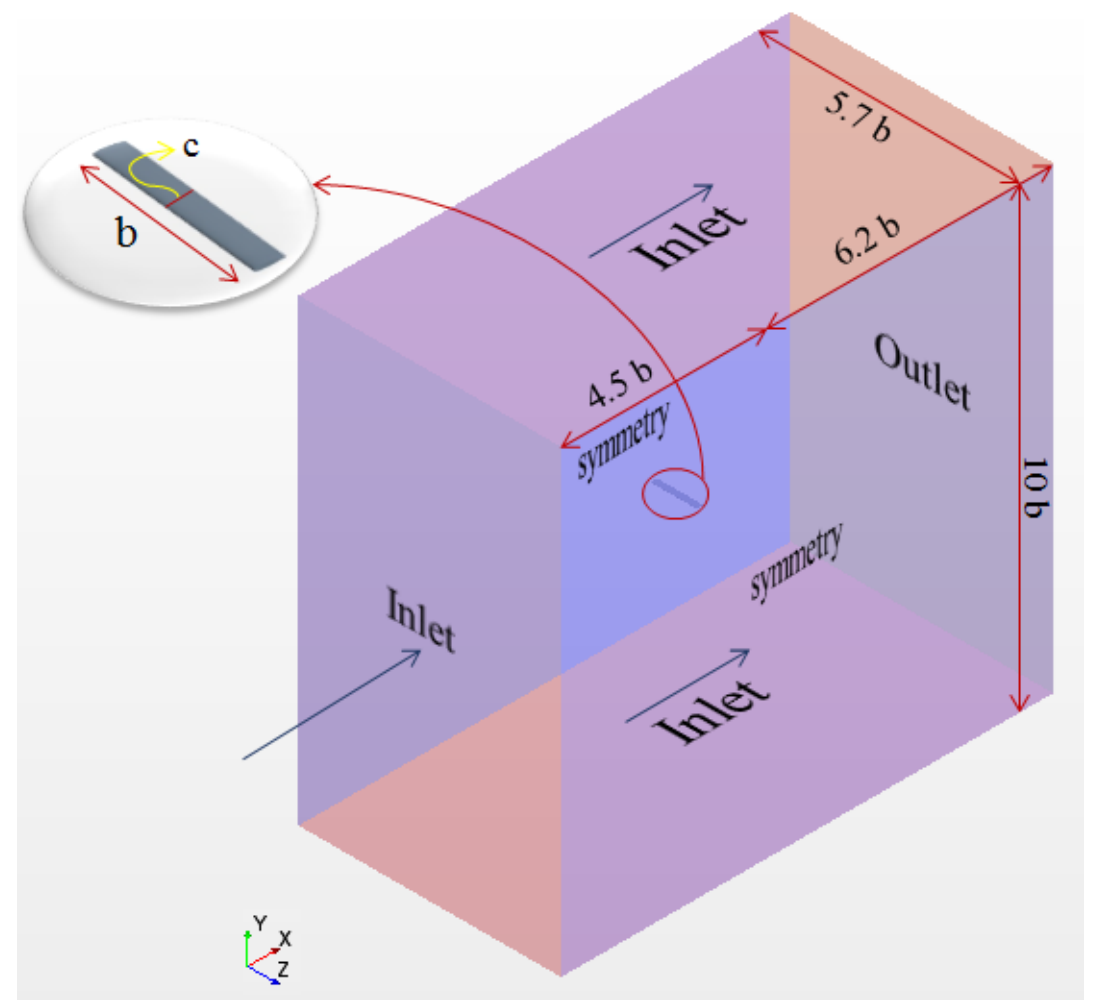

Fig. 2 Computational domain and boundary conditions

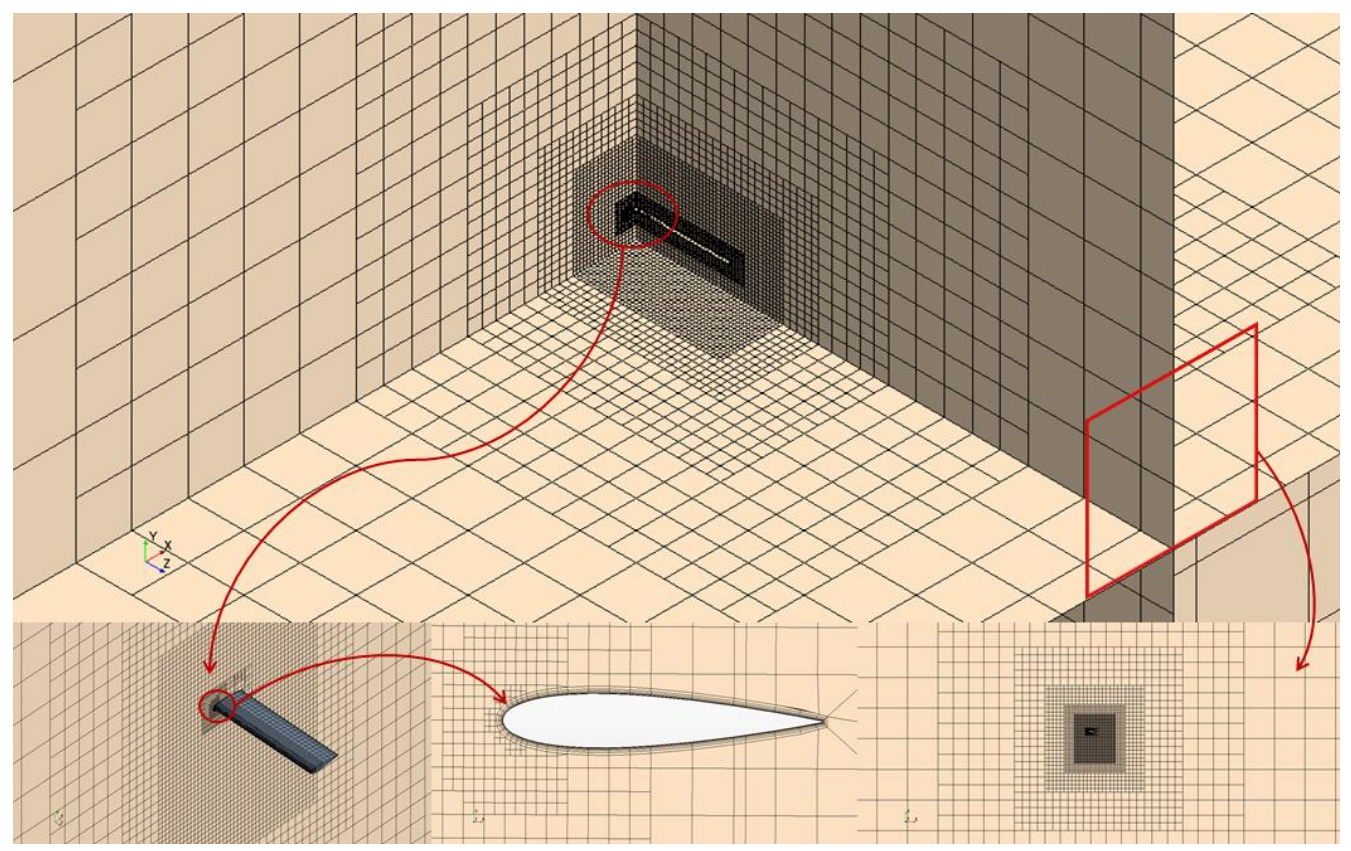

Fig. 3 Domain mesh 


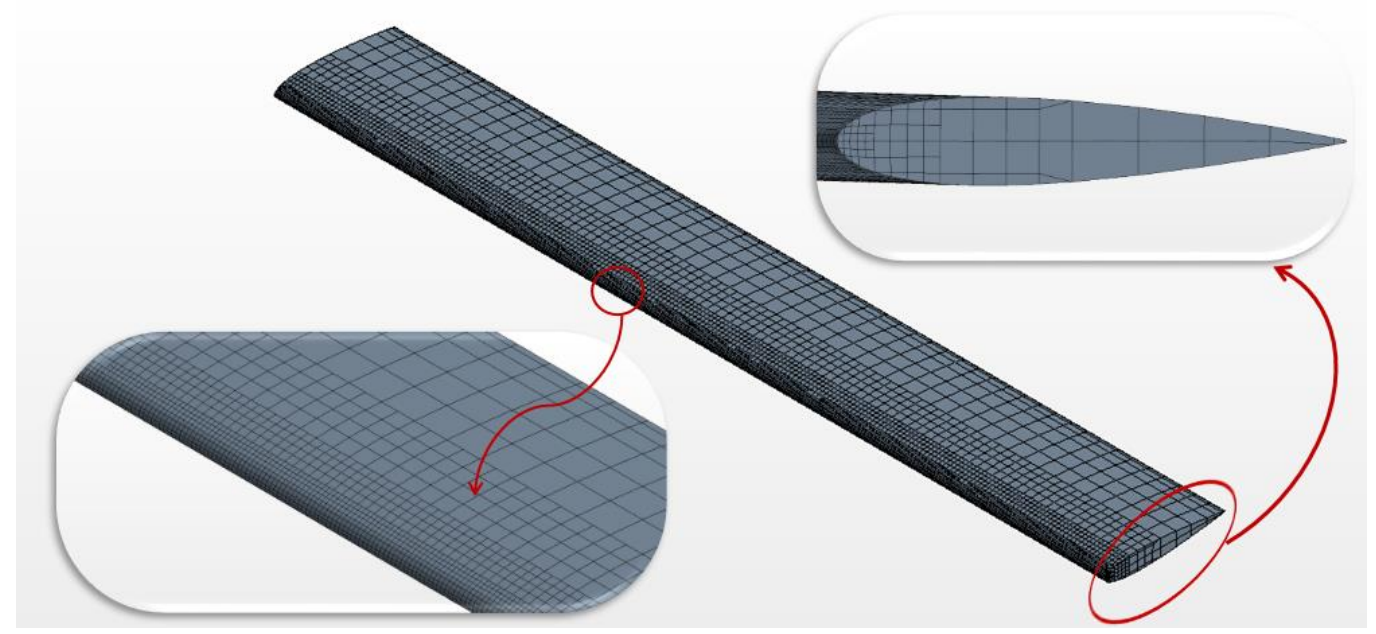

Fig. 4 Hydrofoil mesh

\section{COMOUTATIONAL METHOD}

Recently, many numerical simulations have been performed on fluids hydrodynamic and heat transfer $[32,33]$ using the $\mathrm{k}-\varepsilon$ turbulent model that this simulation have high accuracy. The governing equations of fluid flow include the continuity and Navier-Stokes equations. By applying Reynolds averaging Navier-Stokes (RANS) equations will be obtained:

$$
\begin{gathered}
\frac{\partial \rho}{\partial t}+\frac{\partial}{\partial x_{i}}\left(\rho u_{j}\right)=0 \\
\frac{\partial \rho u_{j}}{\partial t}+\frac{\partial}{\partial x_{j}}\left(\rho u_{i} u_{j}\right)=-\frac{\partial P}{\partial x_{i}}+\frac{\partial}{x_{j}}\left(\tau_{i j}-\rho \overline{u_{\imath}^{\prime} u_{\jmath}^{\prime}}\right)+g_{i}
\end{gathered}
$$

$\overline{\rho^{\prime}{ }^{\prime} u_{\mathrm{j}}{ }^{\prime}}$ represents Reynolds stresses.

Based on turbulent viscosity theory that provides relationship between Reynolds stress terms and velocity gradients, equation (2) is expressed as follows:

$$
\frac{\partial \rho u_{i}}{\partial t}+\frac{\partial}{\partial x_{j}}\left(\rho u_{i} u_{j}\right)=-\frac{\partial P}{\partial x_{i}}+\frac{\partial}{x_{j}}\left[\mu_{e f f}\left(\frac{\partial u_{i}}{\partial x_{j}}+\frac{\partial u_{j}}{\partial x_{i}}\right)\right]+g_{i}
$$

where $\mu_{\text {eff }}$ is the effective viscosity is defined by

$$
\mu \_ \text {eff }=\mu+\mu_{-} t
$$

Two-equation k- $\varepsilon$ model is used to model turbulence flow, in which k represents turbulent kinetic energy of flow, and $\varepsilon$ is dissipation rate of energy. In this model, eddy viscosity (Eq. 5) is related to viscous kinetic energy and dissipation rate:

$$
\mu_{t}=c_{\mu} \rho \frac{k^{2}}{\varepsilon}
$$

where $C_{\mu}$ is constant and $\mathrm{k}, \varepsilon$ are determined by solving the following transport equations:

$$
\begin{gathered}
\frac{\partial(\rho k)}{\partial t}+\frac{\partial}{\partial x_{j}}\left(\rho u_{j} k\right)=\frac{\partial}{\partial x_{j}}\left[\left(\mu+\frac{\mu_{t}}{\sigma_{k}}\right) \frac{\partial k}{\partial x_{j}}\right]+p_{k}-\rho \varepsilon \\
\frac{\partial(\rho \varepsilon)}{\partial t}+\frac{\partial}{\partial x_{j}}\left(\rho u_{j} \varepsilon\right)=\frac{\partial}{\partial x_{j}}\left[\left(\mu+\frac{\mu_{t}}{\sigma_{\varepsilon}}\right) \frac{\partial \varepsilon}{\partial x_{j}}\right]+\frac{\varepsilon}{k}\left(C_{\varepsilon 1} p_{k}-C_{\varepsilon 2} \rho \varepsilon\right)
\end{gathered}
$$

where $C_{\varepsilon 1}, C_{\varepsilon 2}$ and $\sigma_{k}$ are constant values, and $\mathrm{P}_{k}$ is turbulence generation due to viscous forces. 


\section{MOTION ANALYSIS OF OSCILLATING HYDROFOIL}

The oscillating hydrofoil's motion is known to be coupled with heaving and pitching along its rotating axis $x_{p}$. The motion for the oscillating hydrofoil of heave and pitch motions are represented as follows:

$$
\begin{gathered}
h(t)=H_{0} \sin (2 \pi f t+\phi) \\
\theta(t)=\theta_{0} \sin (2 \pi f t)
\end{gathered}
$$

where $H_{0}$ is the heaving amplitude, $\theta_{0}$ is pitching amplitude, $f$ is oscillating frequency, and $\phi$ is the phase angle between the heaving and pitching motions.

According to the hydrofoil motion feature and upstream flow velocity conditions, the angle of attack (AoA) is expressed at the pitching axis of the upstream hydrofoil, which is expressed by:

$$
\alpha(t)=\theta(t)-\arctan \left(\frac{V_{Y}(t)}{U_{\infty}}\right)
$$

where $V_{Y}$ and $U_{\infty}$ are instantaneously heaving velocity and inflow velocity, respectively.

The dimensionless reduced frequency $f^{*}$ is defined as follows:

$$
f^{*}=\frac{f c}{U_{\infty}}
$$

Having the instantaneous AoA and inflow velocity, the instantaneous lift, drag and moment can be determined. Then, the coefficients of the lift, drag and moment are defined as follows:

$$
\begin{aligned}
C_{L}(t) & =\frac{L(t)}{0.5 \rho U_{\infty}^{2} b c} \\
C_{D}(t) & =\frac{D(t)}{0.5 \rho U_{\infty}^{2} b c} \\
C_{M}(t) & =\frac{M(t)}{0.5 \rho U_{\infty}^{2} b c}
\end{aligned}
$$

The lift is perpendicular to the inflow and drag is in-line direction of the inflow. So, horizontal and vertical forces can be found in X-direction and in Y-direction, respectively (as shown in Fig.5). Here, the instantaneous horizontal and vertical forces coefficients are expressed as follows:

$$
\begin{aligned}
C_{X}(t) & =\frac{F_{X}(t)}{0.5 \rho U_{\infty}^{2} b c} \\
C_{Y}(t) & =\frac{F_{Y}(t)}{0.5 \rho U_{\infty}^{2} b c}
\end{aligned}
$$

The instantaneous power extraction $P(t)$ of hydrofoil are obtained by heaving and pitching power which are expressed as:

$$
P(t)=P_{Y}(t)+P_{\theta}(t)=F_{Y}(t) \cdot V_{Y}(t)+M(t) \cdot \omega(t)
$$

The coefficients of the power are defined as follows:

$$
\begin{aligned}
C_{P_{Y}}(t) & =\frac{P_{Y}(t)}{0.5 \rho U_{\infty}^{3} b c} \\
C_{P_{\theta}}(t) & =\frac{P_{\theta}(t)}{0.5 \rho U_{\infty}^{3} b c} \\
C_{P}(t) & =\frac{P(t)}{0.5 \rho U_{\infty}^{3} b c}
\end{aligned}
$$

The total average extraction power $\bar{P}$ can be expressed as: 


$$
\bar{P}=\frac{1}{T} \int_{t}^{t+T} P(t) d t
$$

The average power coefficient over one cycle is expressed as:

$$
\bar{C}_{P}=\frac{1}{T} \int_{t}^{t+T} C_{P}(t) d t
$$

The power efficiency of a hydrofoil is expressed as:

$$
\eta=\frac{\bar{P}}{P_{a}}
$$

where $P_{a}$ is total energy available in the inflow velocity, which is expressed as:

$$
P_{a}=0.5 \rho U_{\infty}^{3} b d
$$

where $d$ is the total extend of the hydrofoil vertical motion and $\rho$ is the density of water.

Fig.5 is illustrated the generated lift and drag during the coupled heave and pitch motions at the one cycle.

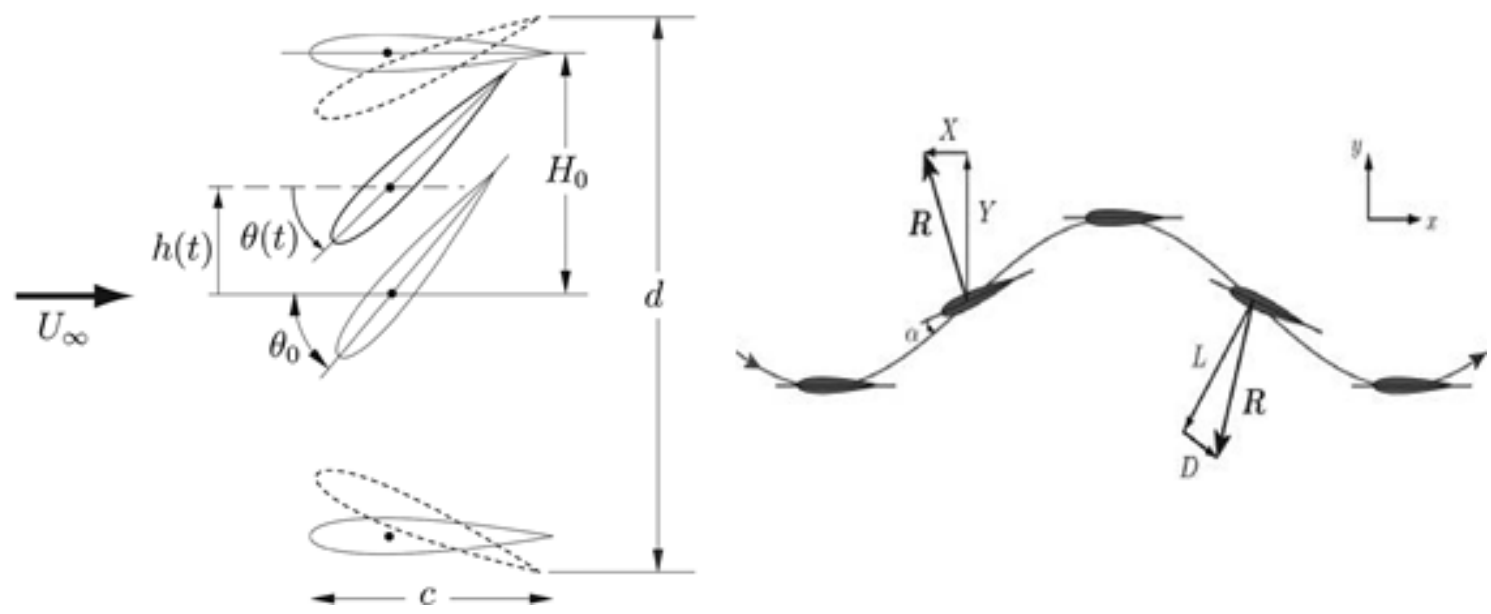

Fig. 5 Lift and drag during heave and pitch motions $[15,17]$

\section{RESULTS AND DISCUSSION}

\subsection{Mesh independence}

The computational method error reduces as the mesh grows smaller, and the computation time rises dramatically. Seven numbers of mesh have been used to compute the efficiency of the oscillating hydrofoil with the section of NACA0015 and a span of $1.68 \mathrm{~m}$. Furthermore, the reduced frequency and flow velocity used for mesh independence investigation are 0.05 and $1.7 \mathrm{~m} / \mathrm{s}$, respectively. In order to independence the results to the mesh and obtain the appropriate accuracy. As can be illustrated in Fig.6, while the number of meshes rises, there would be little improvement in the results thus, to save on computing costs, the number of 405628 meshes was used.

\subsection{Validation}

For 3D rectangular hydrofoil of the NACA0015 with a chord $0.22 \mathrm{~m}$ and span $0.65 \mathrm{~m}$ (aspect ratio is 3 ), the numerical results of the energy extraction efficiency (power efficiency) are compared with experimental data, $[34,35]$ as given in Table 2 . Here, the inflow velocity is 1.7 $\mathrm{m} / \mathrm{s}$ and different reduced frequencies of $0.05,0.073,0.086$, and 0.11 . Furthermore, as can be seen in Fig.7, the numerical results of the vertical and horizontal forces coefficients $\left(C_{X}, C_{Y}\right)$ are compared to the numerical results carried out by [17] at the inflow velocity of $2.014 \mathrm{~m} / \mathrm{s}$ 
and reduced frequency of 0.117 . Expectation on this figure, shows that the present results are good agreement with Kinsey and Dumas results.

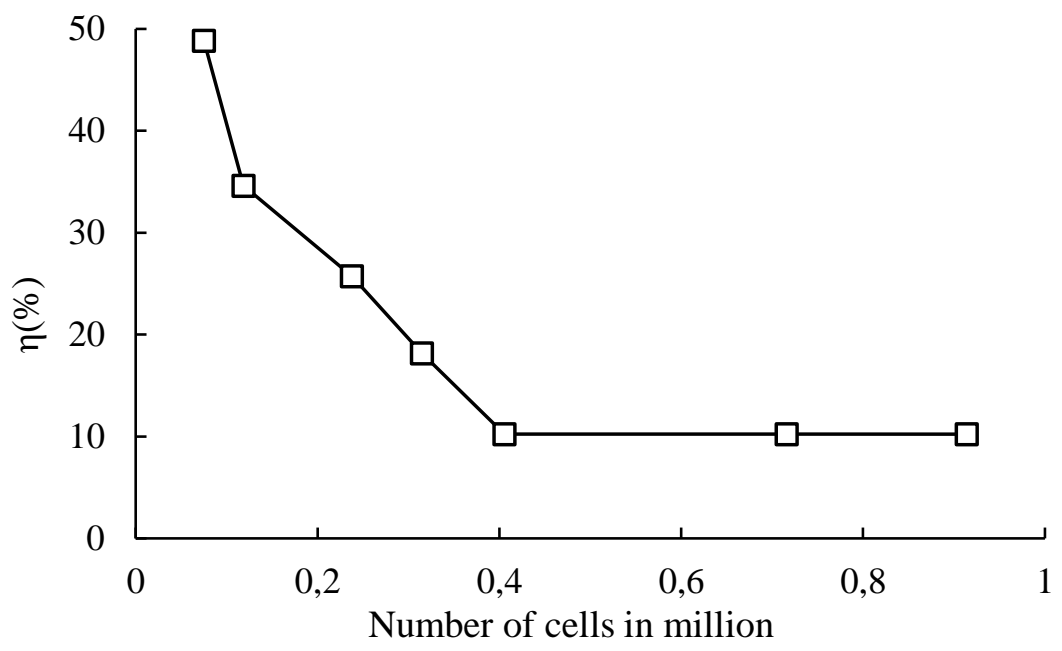

Fig. 6 Mesh independence at the reduced frequency of 0.05 and the flow velocity of $1.7 \mathrm{~m} / \mathrm{s}$

Table 2. Comparison of power efficiency

\begin{tabular}{cccc}
\hline \multicolumn{3}{c}{$\boldsymbol{U}_{\infty}=\mathbf{1 . 7} \mathbf{~ m} / \boldsymbol{s}$} \\
\hline $\boldsymbol{f}^{*}$ & $\eta_{\text {(Present results) }}$ & $\eta_{\text {(EXP) }}$ & Error (\%) \\
\hline 0.050 & 10.2273 & 9.63203 & 6.18011 \\
0.073 & 17.5701 & 17.1537 & 2.42746 \\
0.086 & 19.7346 & 19.3723 & 1.87019 \\
0.110 & 19.1717 & 18.9935 & 0.93821 \\
\hline
\end{tabular}
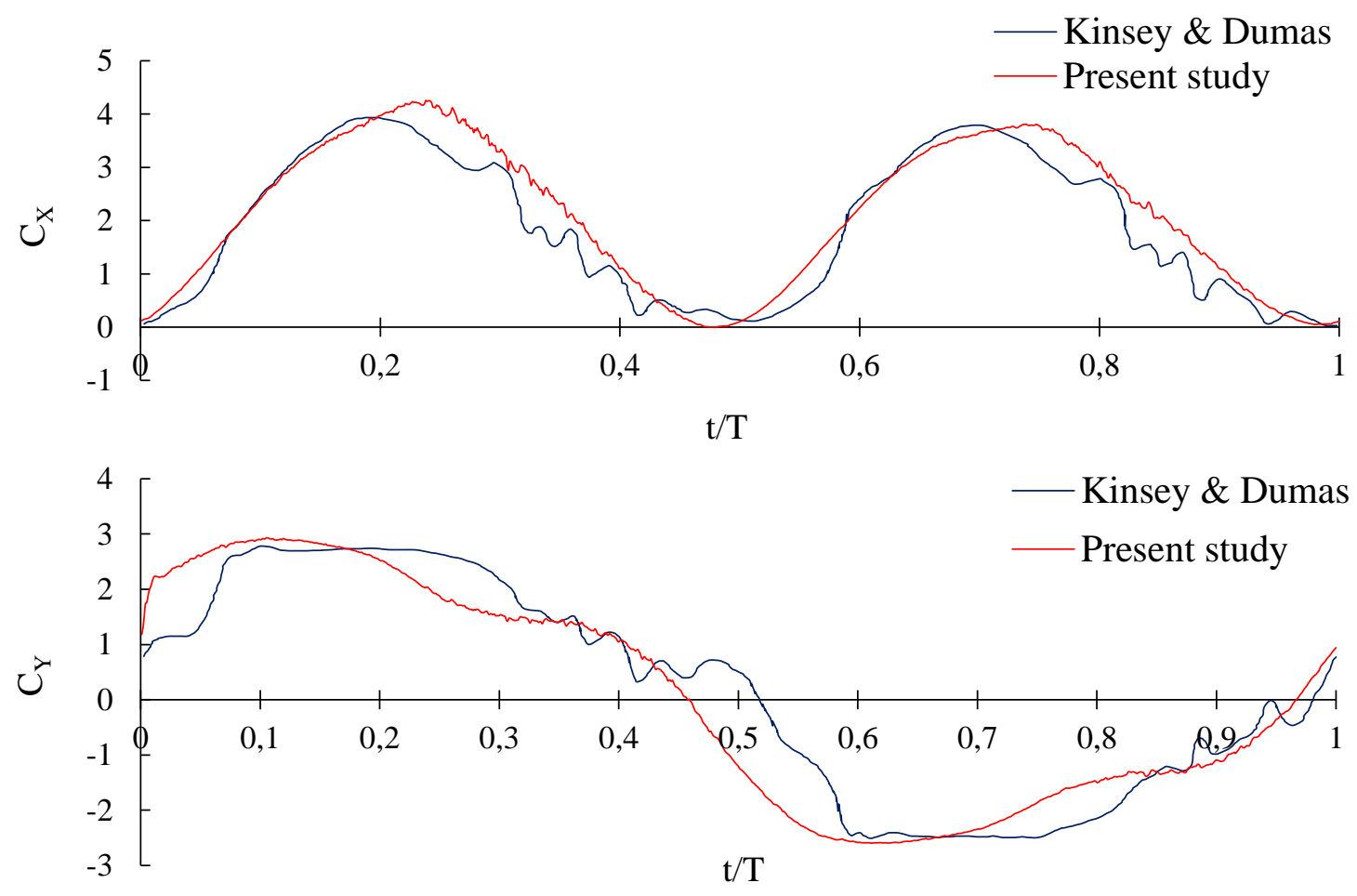

Fig. 7 Comparison of horizontal and vertical coefficients $\left(C_{X}, C_{Y}\right)$ 


\subsection{Numerical results}

The lift and drag coefficient at the flow velocities of 1 and $2 \mathrm{~m} / \mathrm{s}$ under different reduced frequencies $f^{*}(0.06,0.12$, and 0.18$)$ are shown in Fig.8. It can be noted from Fig.8 that at the velocity of $1 \mathrm{~m} / \mathrm{s}$ the maximum peak of curves at all of the $f^{*}$ are higher than the maximum peak of curves at the velocity of $2 \mathrm{~m} / \mathrm{s}$. So, the same result of the drag coefficient can be said for the lift coefficient.

Fig.9 shows the dimensionless horizontal component of hydrodynamic force $\left(C_{X}\right)$, the vertical component of a hydrodynamic force $\left(C_{Y}\right)$ and moment coefficients $\left(C_{M}\right)$ at the inflow velocities of 1 and $2 \mathrm{~m} / \mathrm{s}$ and different reduced frequencies $\left(f^{*}\right)$ of $0.06,0.12$, and 0.18 . From both of $C_{X}$ plot, the numerical values in $f^{*}=0.18$ at the one cycle are higher than numerical values in the $f^{*}=0.12$ and as a result, the numerical values in $f^{*}=0.12$ are higher than $f^{*}=$ 0.06 data. So, for $C_{Y}$ plot can be said the behavior of the curves at all of the reduced frequencies are same to $C_{L}$ plot. About the $C_{M}$ can be said the maximum peak of curves at all of $f^{*}$ at the velocity of $1 \mathrm{~m} / \mathrm{s}$ are higher than the maximum peak of curves at the velocity of $2 \mathrm{~m} / \mathrm{s}$.

Fig.10 is presented the $C_{P_{Y}}, C_{P_{\theta}}$ and $C_{P}$ at the flow velocities of 1 and $2 \mathrm{~m} / \mathrm{s}$ and different $f^{*}$ $(0.06,0.12$, and 0.18$)$. It can be observed from plots that the positive peaks of $C_{P_{Y}}$ curves are near to $\mathrm{t} / \mathrm{T}$ of 0.2 and 0.7 and negative peaks of curves are near to t/T of 0.45 and 0.95 and also for $C_{P_{\theta}}$ the positive peaks are near to $\mathrm{t} / \mathrm{T}$ of $0,0.4$ and 0.9 and the negative peaks are on the ranges of 0.2 to 0.4 and 0.7 to 0.9 . The $C_{P}$ curves are the sum of the $C_{P_{Y}}$ and $C_{P_{\theta}}$.

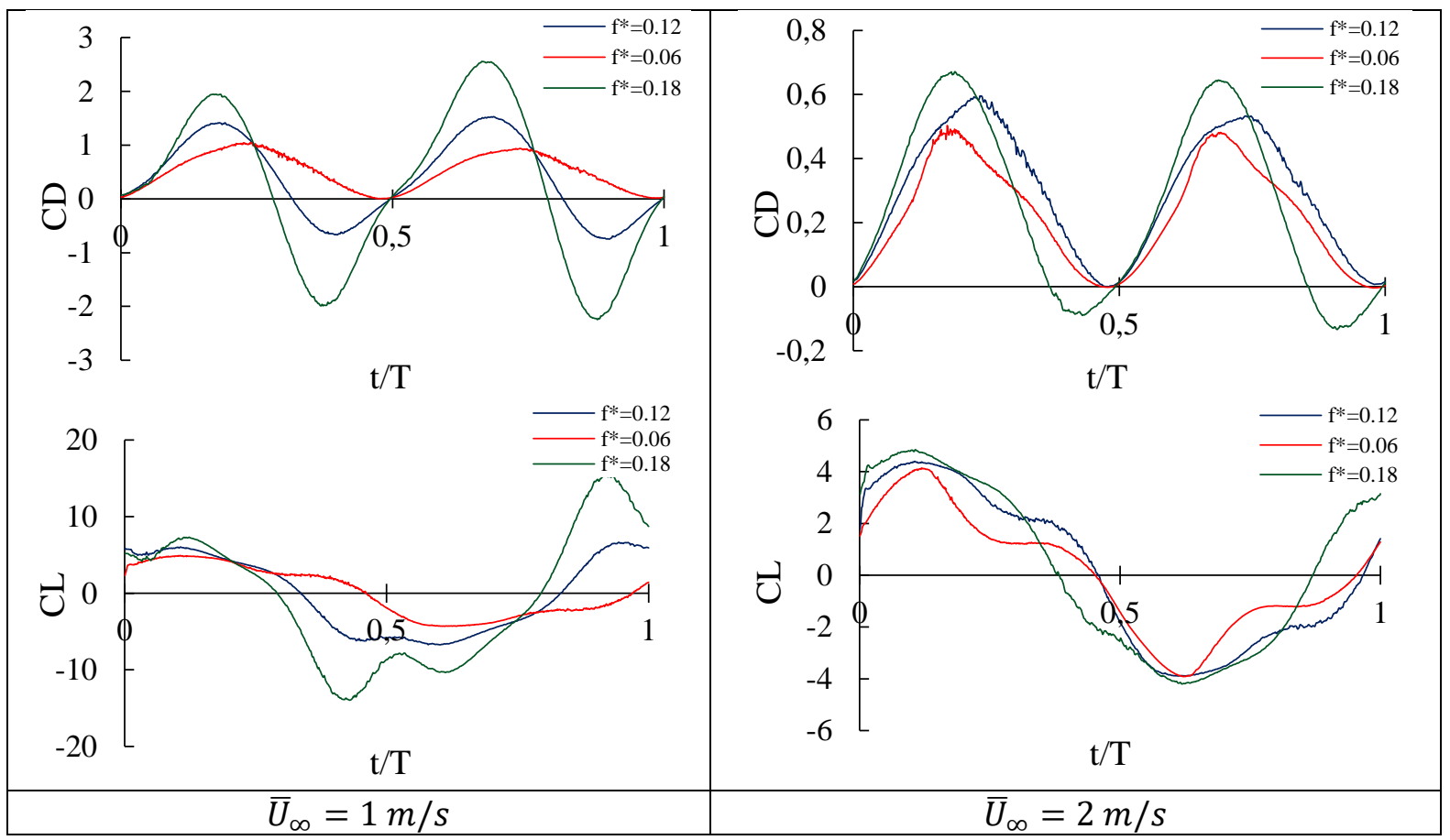

Fig. 8 Lift and drag coefficients $\left(\mathrm{C}_{\mathrm{L}}\right.$, and $\left.\mathrm{C}_{\mathrm{D}}\right)$ under different reduced frequencies.

Time history of the vorticity and wake field contours around the oscillating hydrofoil at the flow velocities of $1 \mathrm{~m} / \mathrm{s}$ and $2 \mathrm{~m} / \mathrm{s}$ in $f^{*}$ of 0.12 during one cycle of rotation and translation motions is shown in Fig.11. It can be noted from the hydrodynamic point of view, the uniform flow is entered to the hydrofoil and then due to changes the AoA and heave motion different states of the vortex are appeared around the trailing edge. The maximum velocity of turbulent flow occurred at $\mathrm{t} / \mathrm{T}=0.2$ because in this time hydrofoil has a maximum AoA (angle of $75^{\circ}$ ). This phenomenon causes that the hydrofoil has a maximum lift and maximum drag and also maximum horizontal and vertical component forces during the oscillating motion. The results 
show when these states have occurred the hydrofoil efficiency increases. Also, it can be seen this state at $\mathrm{t} / \mathrm{T}=0.7$ by similar reasons mentioned.

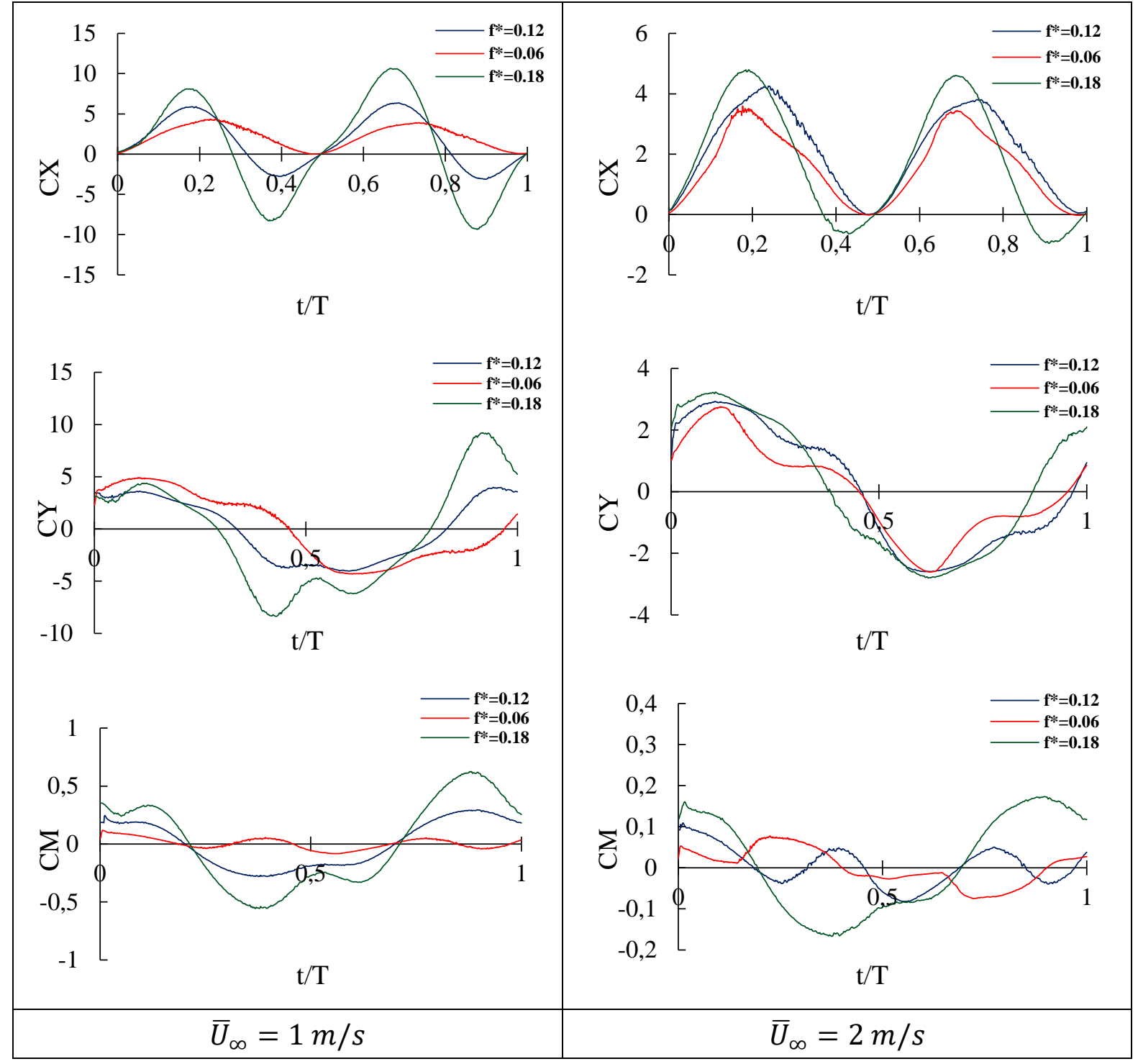

Fig. 9 Results of the $C_{X}, C_{Y}$, and $C_{M}$ under different reduced frequencies.

However, the specificity of three-dimensional flow over a hydrofoil at large vorticity, the corresponding spreading of the boundary layer along the span towards the hydrofoil end sections and the flow separation at these sections cause the increase of drag as well as to have an adverse effect on the oscillating hydrofoil controllability and stability.

Time history of the pressure distribution and streamline contours around the oscillating hydrofoil at the inflow velocities of 1 and $2 \mathrm{~m} / \mathrm{s}$ in $f^{*}$ of 0.12 during one cycle of rotation and translation motion is presented in Fig.12. According to the figure, it can be found that the maximum pressure difference between the up face and down face of the hydrofoil, occurred at $\mathrm{t} / \mathrm{T}=0.2$ and $\mathrm{t} / \mathrm{T}=0.8$ because the AoA is maximum. For this reason and due to the $C_{X}$ plot, the maximum horizontal thrust is at the time of 0.2 and also, between the time of $0.7-0.8$. Furthermore, the streamlines show that the inflow velocity at the leading edge is very low because the event of the flow separation has arisen near this area (from $\theta=20^{\circ}$ to $\theta=75^{\circ}$ ), and with an increased AoA of the hydrofoil during the oscillation, the flow separation increases more rapidly. This is more clearly exhibited for $\theta<20^{\circ}$ where the hydrofoil has been returned 
to the initial state, can be seen as the flow separation and major vorticity near to trailing edge. The above applies to both states of $U_{\infty}=1 \mathrm{~m} / \mathrm{s}$ and $U_{\infty}=2 \mathrm{~m} / \mathrm{s}$.

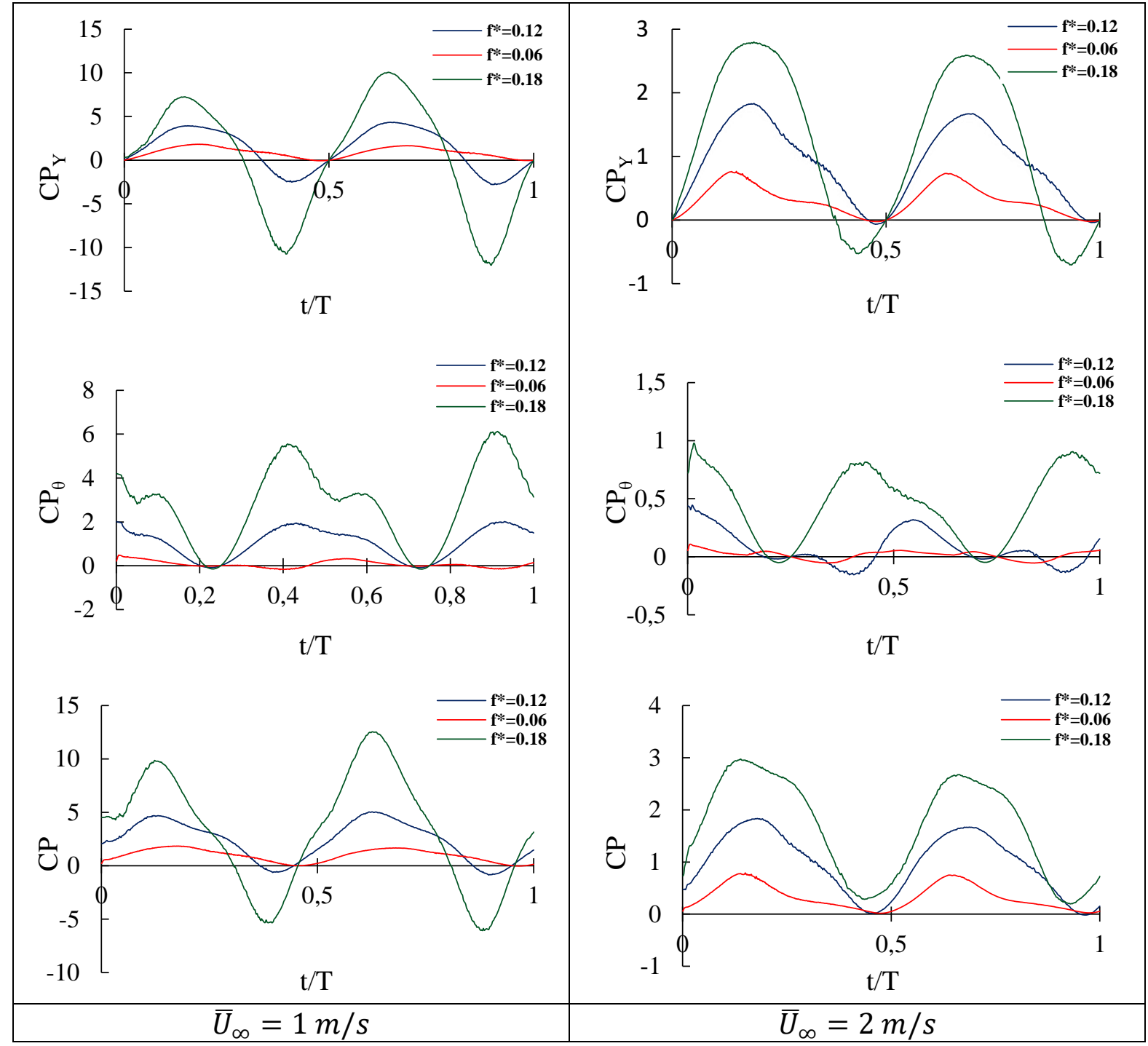

Fig. 10 Results of the $C_{P_{Y}}, C_{P_{\theta}}$ and $C_{P}$ under different reduced frequencies.

The average values of hydrodynamic parameters during one motion-cycle at the three reduced frequencies of $0.06,0.12$, and 0.18 and two velocities of 1 and $2 \mathrm{~m} / \mathrm{s}$ are given in Table 3 and Table 4. As shown in Table 3, with increasing $f^{*}$ the parameters of $\bar{C}_{X}$ and $\bar{C}_{D}$ are diminished and the $\bar{C}_{P_{\theta}}$ and $\bar{C}_{P}$ are increased. Regarding to the parameters of $\bar{C}_{L}, \bar{C}_{Y}$ and $\bar{C}_{M}$, with increasing $f^{*}$ these parameters decrease and then increase but the $\bar{C}_{P_{Y}}$ is obtained in reverse.

According to Table 4 with increasing $f^{*}$ all parameters are increased except to $\bar{C}_{X}$ and $\bar{C}_{D}$ which increase and then decrease. The comparing between parameters of $\bar{C}_{P_{\theta}}, \bar{C}_{P_{Y}}$ and $\bar{C}_{P}$ which play an important role in the performance of the system as Fig. 13 demonstrates. Table 5 is given the power efficiency at three reduced frequencies $(0.06,0.12$, and 0.18$)$ and at the inflow velocities of $1 \mathrm{~m} / \mathrm{s}$ and $2 \mathrm{~m} / \mathrm{s}$. As shown in this table, with increasing the reduced frequencies the power efficiency is increased. 


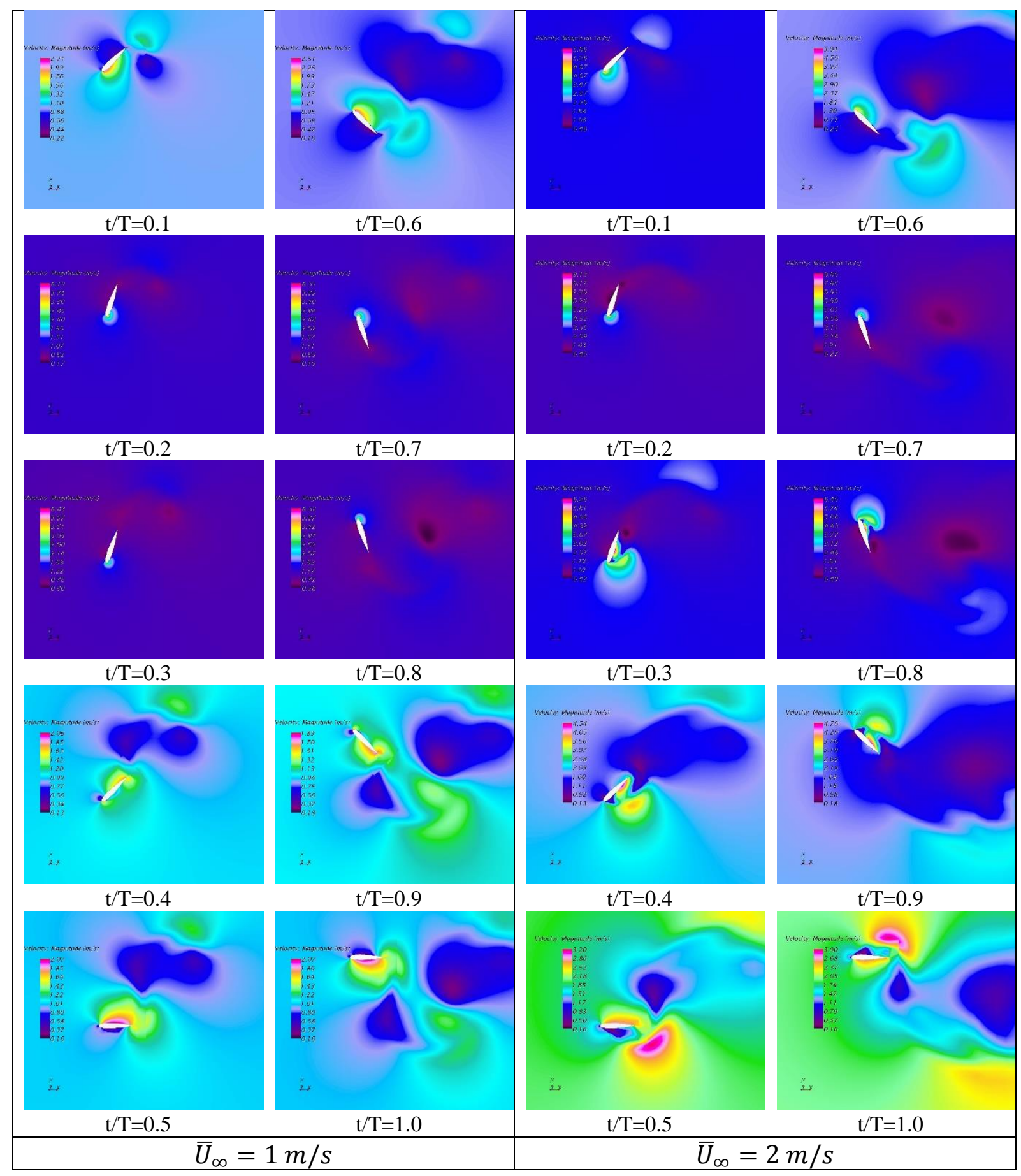

Fig. 11 Time history of the vorticity and wake field around the oscillating hydrofoil

$$
\left(f^{*}=0.12\right)
$$

Table 3. The average of the hydrodynamic coefficients under different reduced frequencies

\begin{tabular}{c|cccccccc}
\hline \multicolumn{8}{c}{$\overline{\mathbf{U}}_{\infty}=\mathbf{1} \boldsymbol{m} / \boldsymbol{s}$} \\
\hline $\mathbf{f}^{*}$ & $\overline{\boldsymbol{C}}_{\boldsymbol{X}}$ & $\overline{\boldsymbol{C}}_{\boldsymbol{Y}}$ & $\overline{\boldsymbol{C}}_{\boldsymbol{M}}$ & $\overline{\boldsymbol{C}}_{\boldsymbol{D}}$ & $\overline{\boldsymbol{C}}_{\boldsymbol{L}}$ & $\overline{\boldsymbol{C}}_{\boldsymbol{P}_{\boldsymbol{\theta}}}$ & $\overline{\boldsymbol{C}}_{\boldsymbol{P}_{\boldsymbol{Y}}}$ & $\overline{\boldsymbol{C}}_{\boldsymbol{P}}$ \\
\hline $\mathbf{0 . 0 6}$ & 2.161041 & 0.072071 & 0.004171 & 0.51865 & 0.120144 & 0.063938 & 0.894497 & 0.958435 \\
$\mathbf{0 . 1 2}$ & 1.578203 & 0.037291 & 0.003494 & 0.37876 & 0.062164 & 1.046331 & 1.252201 & 2.298533 \\
$\mathbf{0 . 1 8}$ & 0.788353 & 0.297193 & 0.010405 & 0.189205 & 0.495422 & 2.850571 & 0.327398 & 3.177969 \\
\hline
\end{tabular}




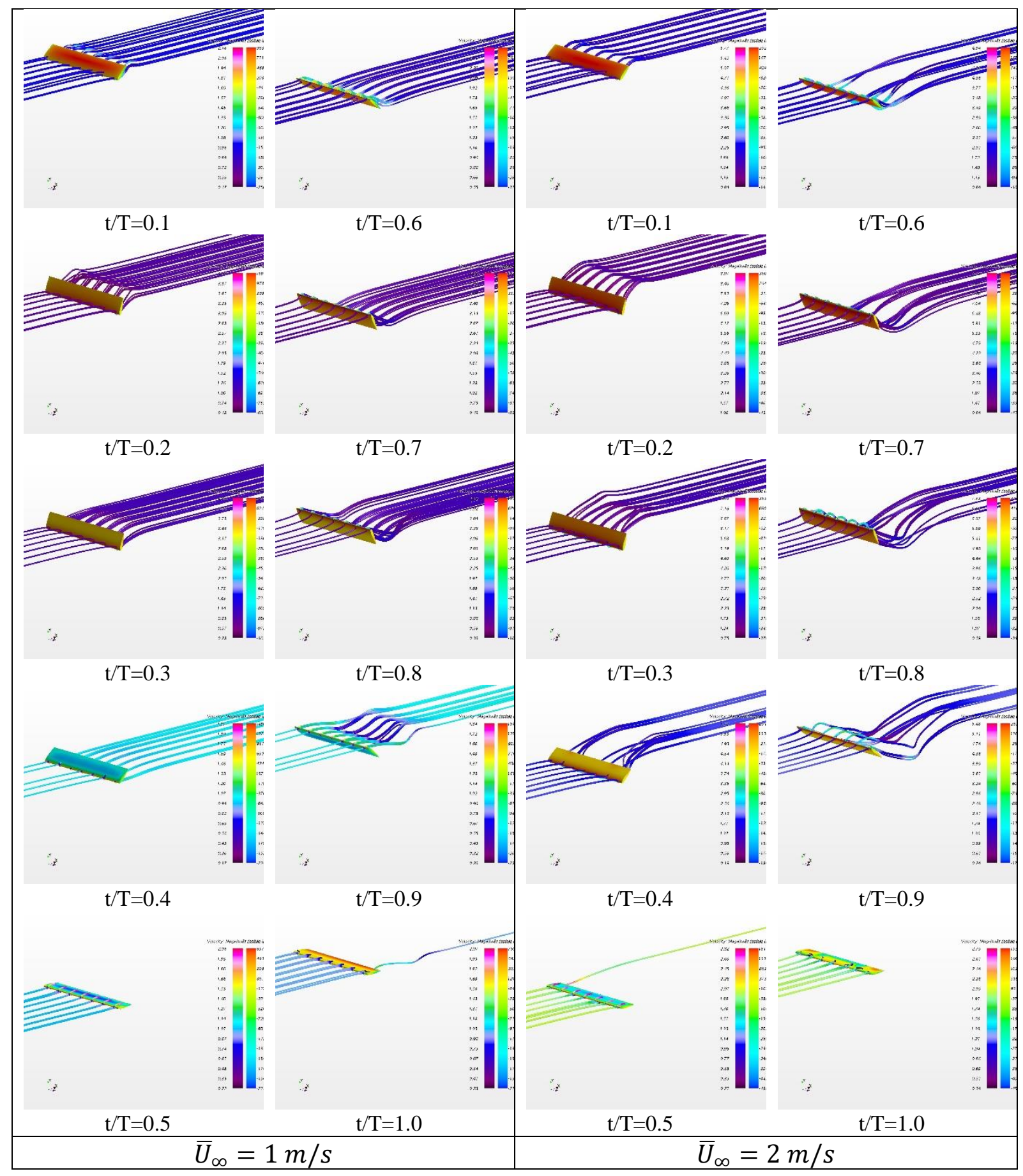

Fig. 12 Time history of the pressure distribution and streamline around the oscillating hydrofoil $\left(f^{*}=0.12\right)$

Table 4. The average of the hydrodynamic coefficients under different reduced frequencies

\begin{tabular}{c|cccccccc}
\hline \multicolumn{8}{c}{$\overline{\mathbf{U}}_{\infty}=\mathbf{2 ~ \boldsymbol { ~ m } / \boldsymbol { s }}$} \\
\hline $\mathbf{f}^{*}$ & $\overline{\boldsymbol{C}}_{\boldsymbol{X}}$ & $\overline{\boldsymbol{C}}_{\boldsymbol{Y}}$ & $\overline{\boldsymbol{C}}_{\boldsymbol{M}}$ & $\overline{\boldsymbol{C}}_{\boldsymbol{D}}$ & $\overline{\boldsymbol{C}}_{\boldsymbol{L}}$ & $\overline{\boldsymbol{C}}_{\boldsymbol{P}_{\theta}}$ & $\overline{\boldsymbol{C}}_{\boldsymbol{P}_{\boldsymbol{Y}}}$ & $\overline{\boldsymbol{C}}_{\boldsymbol{P}}$ \\
\hline $\mathbf{0 . 0 6}$ & 1.60261 & 0.028181 & 0.001486 & 0.224365 & 0.042271 & 0.014977 & 0.309720 & 0.324697 \\
$\mathbf{0 . 1 2}$ & 2.12398 & 0.062286 & 0.003773 & 0.297357 & 0.093429 & 0.066439 & 0.896305 & 0.962744 \\
$\mathbf{0 . 1 8}$ & 1.79217 & 0.177033 & 0.009174 & 0.250904 & 0.265549 & 0.452307 & 1.201641 & 1.653949 \\
\hline
\end{tabular}


Table 5. The power efficiency of oscillating hydrofoil at different reduced frequencies and two inflow velocities.

\begin{tabular}{|c|c|c|}
\hline & $f^{*}$ & $\eta(\%)$ \\
\hline \multirow{3}{*}{$\overline{\mathbf{U}}_{\infty}=\mathbf{1} \mathbf{m} / \mathbf{s}$} & 0.06 & 18.0981 \\
\hline & 0.12 & 42.3863 \\
\hline & 0.18 & 73.6824 \\
\hline \multirow{3}{*}{$\overline{\mathbf{U}}_{\infty}=2 \mathrm{~m} / \mathrm{s}$} & 0.06 & 12.7332 \\
\hline & 0.12 & 37.7546 \\
\hline & 0.18 & 64.8607 \\
\hline
\end{tabular}
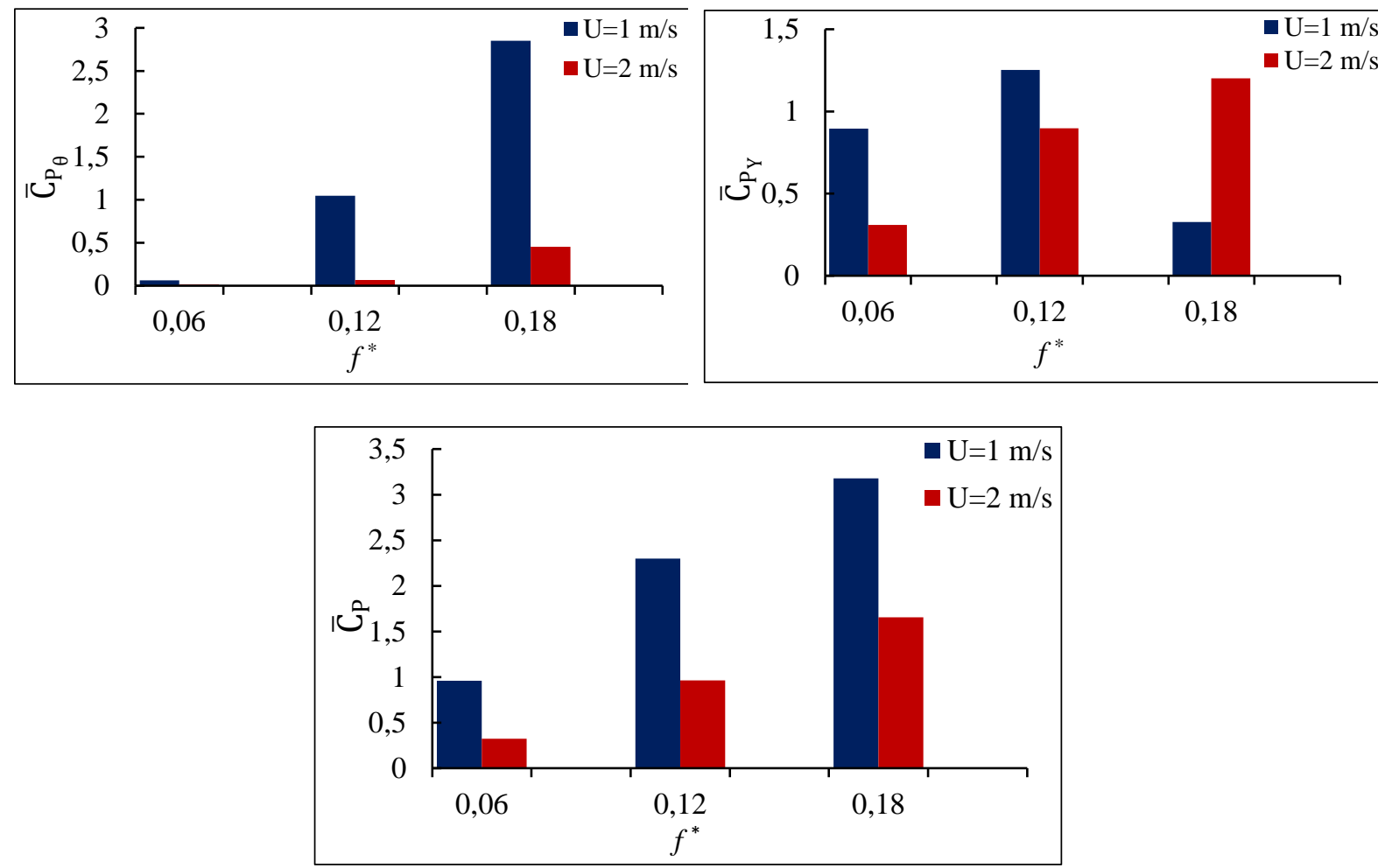

Fig. 13 The comparison between parameters of $\bar{C}_{P_{\theta}}, \bar{C}_{P_{Y}}$, and $\bar{C}_{P}$

\section{CONCLUSION}

This research is numerically investigated on the extracted power by the coupled heaving and pitching oscillating hydrofoil performance. The Star-CCM+ software and realizable k- $\varepsilon$ turbulent model was used. In the present work, the lift and drag coefficients, moment coefficient, power efficiency, pressure distribution, wake field and vorticity around the oscillating hydrofoil (heave and pitch motions) under different reduced frequencies and two inflow velocities are calculated. Based on the numerical result, the following conclusions can be drawn:

- The power efficiency, lift and drag coefficients are compared with experimental data and other numerical results and showed good agreement between them.

- The power coefficient is improved by increasing the reduced frequency.

- With increasing the reduced frequency, the power efficiency is increased at both inflow velocities.

- The maximum value of the lift, drag, horizontal and vertical forces is found at $\mathrm{t} / \mathrm{T}=0.2$ and $\mathrm{t} / \mathrm{T}=0.7$ due to higher AoA. 
- The fields of turbulent flows are affected by erratic and variable velocity. These variations contain transported characteristics such as momentum and energy.

- The computation result allows for a more detailed analysis of the flow field characteristics for the reported lift and drag qualities.

- The data clearly demonstrate that changing the motion frequency with different flow speeds changes the whole flow field over the hydrofoil.

- Flow patterns with varying motion angles may be created by reconstructing with different modes. In this form, the vortex phase characteristics of the flow field at various hydrofoil angles have been investigated.

In some cases, flow separation occurs and causes an inappropriate effect on the oscillating hydrofoil stability. More emphasis will be investigated in the future work.

\section{REFERENCES}

[1] Wang, J., Liu, P., Chin, C., He, G. "Numerical investigation of auto-pitch wing-in-ground effect oscillating foil propulsor", Applied Ocean Research 89, pp. 71 - 84, 2019. DOI: 10.1016/j.apor.2019.05.015

[2] Yan, Y., Avital, E., Williams, J., Cui, J. "CFD analysis for the performance of micro-vortex generator on aerofoil and vertical axis turbine", Journal of Renewable and Sustainable Energy 11 (4), p. 043302, 2019. DOI: $10.1063 / 1.5110422$

[3] Abbasi, A., Ghassemi, H., Molyneux, D. "Numerical analysis of the hydrodynamic performance of HATST with different blade geometries", American Journal of Civil Engineering and Architecture 6 (6), pp. 236 - 241, 2018. DOI: 10.12691/ajcea-6-6-2

[4] Abbasi, A., Ghassemi, H., Molyneux, D. "Power and thrust coefficients of the horizontal axis tidal stream turbine with different twist angles, blade numbers, and section profiles, Scientific", Journals of the Maritime University of Szczecin 57 (129), pp. 11 - 20, 2019. DOI: 10.17402/321

[5] Lee, J., Choi, H., Kim, H.Y. "A scaling law for the lift of hovering insects", Journal of Fluid Mechanics 782, pp. 479 - 490, 2015. DOI: 10.1017/jfm.2015.568

[6] Shyy, W., Aono, H., Chimakurthi, S.K., Trizila, P., Kang, C.K., Cesnik, C.E., Liu, H. "Recent progress in flapping wing aerodynamics and aeroelasticity", Progress in Aerospace Sciences 46 (7), pp. 284 - 327, 2010. DOI: 10.1016/j.paerosci.2010.01.001

[7] Lin, X., Wu, J., Zhang, T. "Performance investigation of a self-propelled foil with combined oscillating motion in stationary fluid", Ocean Engineering 175, pp. 33 - 49, 2019. DOI: 10.1016/j.oceaneng.2019.02.008

[8] Budiyanto, M. A., Syahrudin, M. F., Murdianto, M. A. "Investigation of the effectiveness of a stern foil on a patrol boat by experiment and simulation", Cogent Engineering 7 (1), p. 1716925, 2020. DOI: $10.1080 / 23311916.2020 .1716925$

[9] Iverson, D., Rahimpour, M., Lee, W., Kiwata, T., Oshkai, P. "Effect of chordwise flexibility on propulsive performance of high inertia oscillating-foils", Journal of fluids and structures 91, p. 102750, 2019. DOI: 10.1016/j.jfluidstructs.2019.102750

[10] Cleaver, D. J., Calderon, D. E., Wang, Z., Gursul, I. "Periodically plunging foil near a free surface", Experiments in fluids 54 (3), pp. 1 - 18, 2013. DOI:10.1007/s00348-013-1491-9

[11] Martin, A. K., Anathakrishanan, P., Krishnankutty, P. "Ship hull wake effect on the hydrodynamic performance of a heave-pitch combined oscillating fin, Ships and Offshore Structures", pp. 1 - 11, 2020. DOI: 10.1080/17445302.2020.1777925

[12] Thaweewat, N., Phoemsapthawee, S., Juntasaro, V. "Semi-active flapping foil for marine propulsion", Ocean Engineering 147, pp. 556 - 564, 2018. DOI: 10.1016/j.oceaneng.2017.11.008 
[13] McKinney, W., DeLaurier, J. “The Wingmill: an oscillating-wing windmill”, Journal of energy 5 (2), pp. 109 - 115, 1981. DOI: $10.2514 / 3.62510$

[14] Kinsey, T., Dumas, G., Lalande, G., Ruel, J., Mehut, A., Viarouge, P., Lemay, J., Jean, Y. "Prototype testing of a hydrokinetic turbine based on oscillating hydrofoils", Renewable energy 36 (6), pp. 1710 - 1718, 2011. DOI: 10.1016/j.renene.2010.11.037

[15] Kinsey, T., Dumas, G. "Parametric study of an oscillating airfoil in a power-extraction regime", AIAA journal 46 (6), pp. 1318 - 1330, 2008. DOI: 10.2514/1.26253

[16] Kinsey, T., Dumas, G. "Optimal operating parameters for an oscillating foil turbine at Reynolds number 500,000”, AIAA Journal 52 (9), pp. 1885 - 1895, 2014. DOI: 10.2514/1.J052700

[17] Kinsey, T., Dumas, G. "Computational fluid dynamics analysis of a hydrokinetic turbine based on oscillating hydrofoils", Journal of fluids engineering 134 (2), 2012. DOI: 10.1115/1.4005841

[18] Huxham, G. H., Cochard, S., Patterson, J. "Experimental parametric investigation of an oscillating hydrofoil tidal stream energy converter", In Proceedings of 18th Australasian Fluid Mechanics Conference AFMC, Australasian Fluid Mechanics Society Launceston, Tasmania, pp. 3-7, 2012.

[19] Harding, S. F., Payne, G. S., Bryden, I. G. "Generating controllable velocity fluctuations using twin oscillating hydrofoils: experimental validation", Journal of fluid mechanics 750, pp. 113 123, 2014. DOI: $10.1017 / \mathrm{jfm} .2014 .257$

[20] Shimizu, E., Isogai, K., Obayashi, S. "Multiobjective design study of a flapping wing power generator", Journal of Fluids Engineering 130 (2), 2008. DOI: 10.1115/1.2829580

[21] Simpson, B. J. "Experimental studies of flapping foils for energy extraction, (Doctoral dissertation, Massachusetts Institute of Technology), 2009. http://hdl.handle.net/1721.1/55283

[22] Jones, K., Platzer, M., Jones, K., Platzer, M. "Numerical computation of flapping-wing propulsion and power extraction", In 35th Aerospace Sciences Meeting and Exhibit, 1997, January, p. 826. DOI: $10.2514 / 6.1997-826$

[23] Anderson, J. M., Streitlien, K., Barrett, D. S. "Triantafyllou, M.S. Oscillating foils of high propulsive efficiency", Journal of Fluid mechanics 360, pp. 41 - 72, 1998. DOI: $10.1017 /$ S0022112097008392

[24] Dewey, P. A., Quinn, D. B., Boschitsch, B. M., Smits, A. J. "Propulsive performance of unsteady tandem hydrofoils in a side-by-side configuration", Physics of Fluids 26 (4), p.041903, 2014. DOI: $10.1063 / 1.4871024$

[25] He, M., Veitch, B., Bose, N. "Colbourne, B., Liu, P. A three-dimensional wake impingement model and applications on tandem oscillating foils", Ocean engineering 34 (8-9), pp. 1197 - 1210, 2007. DOI: 10.1016/j.oceaneng.2006.07.002

[26] Giovannetti, L. M., Banks, J., Ledri, M., Turnock, S. R. and Boyd, S. W. "Toward the development of a hydrofoil tailored to passively reduce its lift response to fluid load", Ocean Engineering 167, 2018. DOI: 10.1016/j.oceaneng.2018.08.018

[27] Pourmahdavi, M., Liu, P. "Shallow water effect of tandem flapping foils on renewable energy production", International Journal of Green Energy 16 (14), pp. 1353 - 1362, 2019. DOI: 10.1080/15435075.2019.1671410

[28] Ma, P., Wang, Y., Xie, Y., Han, J., Sun, G., Zhang, J. "Effect of wake interaction on the response of two tandem oscillating hydrofoils", Energy Science \& Engineering 7 (2), pp. 431 - 442, 2019. DOI: $10.1002 /$ ese 3.286

[29] He. G., Mo, W. Gao Y., Zhang Z., Wang J., Wang, W., Liu P, Ghassemi H. "Modification of effective angle of attack on hydrofoil power extraction", Ocean engineering 240, 2021. DOI: 10.1016/j.oceaneng.2021.109919 
[30] Pourmostafa, M., Ghassemi, H., Ghadimi,: P. "Boundary Element Method Applied to the 2D Foil with Oscillating Heave and Pitch Motions", American Journal of Mechanical Engineering 8 (1), pp. 17 - 25, 2020. DOI: 10.12691/ajme-8-1-3

[31] Satwika, N. A., Hantoro, R., Sarwono, S., Nugroho, G. "The experimental investigation and numerical analysis on horizontal axis wind turbine with winglet and pitch variations", Engineering Journal 23 (6), pp. 345 - 360, 2019. DOI: 10.4186/ej.2019.23.6.345

[32] Kutiš, V., Jakubec, J., Paulech, J., Gálik, G., Sedlár, T. "CFD analysis of downcomer of nuclear reactor VVER 440”, Strojnícky časopis - Journal of Mechanical Engineering 66 (2), pp. 55 - 62, 2016. DOI: $10.1515 /$ scjme-2016-0018

[33] Kapilan, N., Gowda, M. M., Manjunath, H. N. "Computational fluid dynamics analysis of an evaporative cooling system", Strojnícky časopis - Journal of Mechanical Engineering 66 (2), pp.117 - 124, 2016. DOI: 10.1515/scjme-2016-0026

[34] Xu, J., Sun, H., Tan, S. "Wake vortex interaction effects on energy extraction performance of tandem oscillating hydrofoils", Journal of Mechanical Science and Technology 30 (9), pp. 4227 -4237, 2016. DOI: $10.1007 / \mathrm{s} 12206-016-0835-9$

[35] Xu, J., Tan, S., Guan, D., Ali, R., Zhang, L. "Energy extraction performance of motionconstrained tandem oscillating hydrofoils", Journal of Renewable and Sustainable Energy 9 (4), p. 044501,2017 . DOI: $10.1063 / 1.4994098$ 